\title{
Local transverse feedback linearization for nested sets
}

\author{
Alireza Doosthoseini and Christopher Nielsen
}

\begin{abstract}
We study a local feedback equivalence problem for a nonlinear control-affine system with two nested, controlled invariant, embedded submanifolds in its state space. The main result of this paper gives necessary and sufficient conditions for (i) the dynamics of the system transversal to the larger submanifold to be linear and controllable and (ii) the system dynamics, restricted to the larger submanifold, and transversal to the smaller submanifold to also be linear and controllable.

Index Terms-Feedback equivalence, partial feedback linearization, nested sets.
\end{abstract}

\section{INTRODUCTION}

One approach a solving sophisticated control problems is to split the problem into prioritized sub-problems and solve them separately. This method is known as hierarchical control design. In [1] the hierarchical control design problem is viewed as the simultaneous stabilization of a chain of closed nested, controlled invariant, sets $S_{1} \supset S_{2} \supset \cdots \supset S_{n}$ in which set $S_{i}$ represents the sub-problem $i$. Set $S_{i}$ being nested in the set $S_{i-1}$ indicates that the sub-problem $i+1$ is solved only if the sub-problem $i$ is solved. For instance, in [2] the hierarchical control design approach is adopted to solve the coordinated path following problem of a multi-agent system of unicycles. There, the coordinated path following problem is decomposed into path following and coordination sub-problems and path following is prioritized over coordination. The objective of hierarchical control design problem is twofold : to design control laws solving each sub-problem independently and to investigate conditions under which the designed control laws solve the main problem when working together. Studies investigating the second aspect include [3], [1]. In this paper we are concerned with the first aspect of hierarchical control design problem.

In this paper we take a feedback equivalence approach that facilitates the control design to locally solve each subproblem. Two control systems are said to be feedback equivalent if there exists a local coordinate and feedback transformation mapping their trajectories to each other. An important subdivision of feedback equivalence problem is called feedback linearization in which feedback equivalence between a nonlinear control system and a controllable linear, or partially linear, system is considered. Seminal works in this field include [4], [5].

Partial feedback linearization has been adopted to solve set stabilization problems. In [6] a single-input control system

Supported by the Natural Sciences and Engineering Research Council of Canada (N.S.E.R.C.)

A. Doosthoseini and C. Nielsen are with the Department of Electrical and Computer Engineering, University of Waterloo, 200 University Avenue West, Waterloo, Ontario, Canada, N2L 3G1. \{adoostho, cnielsen\}@uwaterloo.ca and a periodic orbit of the system is considered and feedback linearization is employed to make the dynamics transversal to the orbit linear and controllable. This motivates the name transverse feedback linearization coined for the first time in that study. In [7] the local transverse feedback linearization problem is formulated and solved for a multi-input control affine system with an embedded controlled invariant submanifold in its state space. The problem entails finding conditions for local feedback equivalence to a control system whose dynamics transversal to the target set are linear and controllable.

This paper generalizes local transverse feedback linearization to two, nested, controlled invariant submanifolds. Given a smooth, time-invariant, nonlinear control-affine system and two nested, controlled-invariant, embedded submanifolds $S_{1}$ and $S_{2}$ the main problem in this paper is to find a local diffeomorphism and feedback transformation, valid in a neighbourhood of a point on $S_{2}$, that bring the control system into a normal form whose dynamics transversal to $S_{1}$ as well as dynamics restricted to $S_{1}$ and transversal to $S_{2}$ are both linear and controllable. The solution of this problem simplifies designing controllers for local stabilization of $S_{1}$ and local stabilization of $S_{2}$ relative to $S_{1}$. Theorem IV.1 presents geometric necessary and sufficient conditions for the problem to be solvable. ${ }^{1}$

\section{A. Notation}

If $k$ is a natural number, $\mathbf{k}:=\{0, \ldots, k\}$. If $U$ is an open connected subset of $\mathbb{R}^{n}$, then $\operatorname{Diff}(U)$ is the collection of diffeomorphisms with domain $U$. If $F: M \rightarrow N$ is a map between manifolds then $\mathrm{d} F(x): T_{x} M \rightarrow T_{F(x)} N$ denotes its differential. If $M$ and $N$ are vector spaces, then $\mathrm{d} F(x)$ is the Jacobian matrix of $F$ evaluated at $x$. If $F: M \rightarrow N$ is a diffeomorphism between two manifolds, and if $v$ is a vector field on $M$, then the differential of $F$ can be used to define a vector field on $N$ by means of the push-forward map $F_{\star}$, defined as $F_{\star} v(q)=\left.\left(d F_{p} v(p)\right)\right|_{p=F^{-1}(q)}$. If $D$ is a nonsingular distribution on a manifold $M, D^{\perp}$ is the orthogonal complement of $D$ obtained from the orthogonal structure on the tangent bundle $T M$. The non-singular distribution $D^{\perp}$ is a subbundle of $T M$ and satisfies, for each $p \in M$, $T_{p} M=D(p) \oplus D^{\perp}(p)$. Let $\operatorname{inv}(D)$ denote the involutive closure of $D$. We denote by $I_{m}$ the $m \times m$ identity matrix while $0_{m \times n}$ denotes an $m \times n$ matrix of zeros. Let $\mathrm{GL}(n, \mathbb{R})$ denote the group of non-singular $n \times n$ matrices with real entires.

\footnotetext{
${ }^{1}$ This paper contains portions of the extended manuscript [8].
} 


\section{LOCAL NESTED TRANSVERSAL FEEDBACK LINEARIZATION}

Consider a control-affine system

$$
\dot{x}=f(x)+\sum_{i=1}^{m} g_{i}(x) u_{i}=: f(x)+g(x) u
$$

where $x \in \mathbb{R}^{n}$ denotes the state, $u=\left(u_{1}, \cdots, u_{m}\right) \in \mathbb{R}^{m}$ is the control input, and $f: \mathbb{R}^{n} \rightarrow \mathbb{R}^{n}$ and $g_{i}: \mathbb{R}^{n} \rightarrow \mathbb{R}^{n}$, $i \in\{1, \cdots, m\}$, are smooth. To (1) we associate the family of distributions

$$
G_{i}:=\operatorname{span}\left\{a d_{f}^{j} g_{k}: 0 \leq j \leq i, 1 \leq k \leq m\right\} .
$$

The vectors $g_{1}(x), \ldots, g_{m}(x)$ are assumed to be linearly independent at each $x \in \mathbb{R}^{n}$, i.e., $\operatorname{dim}\left(G_{0}(x)\right)=m$. Along with (1), we are also given two embedded submanifolds $S_{1} \subset \mathbb{R}^{n}$ and $S_{2} \subset \mathbb{R}^{n}$ with $s_{1}:=\operatorname{dim}\left(S_{1}\right), s_{2}:=\operatorname{dim}\left(S_{2}\right)$. The following assumption is made throughout this paper.

Assumption 1. The sets $S_{1}$ and $S_{2}$ are closed controlledinvariant embedded submanifolds for (1) and $S_{1} \supset S_{2}$.

Problem 1. (Local nested transversal feedback linearization) Given (1), nested sets $S_{1} \supset S_{2}$ satisfying Assumption 1 and a point $\bar{x} \in S_{2}$, find, if possible, a diffeomorphism $\Xi: U \rightarrow \Xi(U) \subset \mathbb{R}^{s_{2}} \times \mathbb{R}^{s_{1}-s_{2}} \times \mathbb{R}^{n-s_{1}}, x \mapsto(\zeta, \mu, \xi)$, and a regular feedback transformation $(\alpha, \beta), u \mapsto\left(v^{\|}, v^{\|, \pitchfork}, v^{\pitchfork}\right)$ valid in a neighbourhood $U \subseteq \mathbb{R}^{n}$ of $\bar{x}$, such that (1) is feedback equivalent to

$$
\begin{aligned}
& \dot{\zeta}=f_{1}+g_{11} v^{\|}+g_{12} v^{\|, \pitchfork}+g_{13} v^{\pitchfork} \\
& \dot{\mu}=A \mu+B v^{\|, \pitchfork}+f_{2}+g_{21} v^{\|}+g_{22} v^{\|, \pitchfork}+g_{23} v^{\pitchfork} \\
& \dot{\xi}=A^{\pitchfork} \xi+B^{\pitchfork} v^{\pitchfork}
\end{aligned}
$$

where $f_{1}, f_{2}, g_{11}, g_{12}, g_{13}, g_{21}, g_{22}, g_{23}$ are functions of $(\zeta, \mu, \xi)$,

$$
\begin{aligned}
& \Xi\left(S_{1} \cap U\right)=\{(\zeta, \mu, \xi) \in \Xi(U): \xi=0\}, \\
& \Xi\left(S_{2} \cap U\right)=\{(\zeta, \mu, \xi) \in \Xi(U): \xi=0, \mu=0\},
\end{aligned}
$$

$f_{2}(\zeta, \mu, 0)=0, g_{21}(\zeta, \mu, 0)=0, g_{22}(\zeta, \mu, 0)=0$, the pairs $(A, B)$ and $\left(A^{\pitchfork}, B^{\dagger}\right)$ are controllable, and the matrices $B$ and $B^{\dagger}$ are full rank.

Problem 1 seeks a coordinate and feedback transformation valid in a neighbourhood of $\bar{x}$ which generates a normal form with two types of decompositions. First the dynamics are decomposed into three subsystems; namely the $\xi-, \mu-$, and $\zeta$-subsystems. We call the $\xi$-subsystem the transversal dynamics to $S_{1}$. This is motivated by the fact that, in the light of (4a), stabilizing $S_{1} \cap U$ is equivalent, under mild assumptions, to stabilizing the $\xi$-subsystem. We call the $\mu$ subsystem the transversal dynamics of $S_{2}$, restricted to $S_{1}$. Similarly, this name is motivated by the fact that, in the light of (4b), stabilizing $S_{2} \cap U$ relative to $S_{1} \cap U$ is equivalent, under mild assumptions, to stabilizing the $\mu$-subsystem when $\xi=0$. The $\zeta$-subsystem is called the tangential dynamics to $S_{2}$ because when $\xi=0$ and $\mu=0$, the $\zeta$ dynamics govern the system's evolution on $\Xi\left(S_{2} \cap U\right)$.
The second type of decomposition is in the original $m$ inputs. They are partitioned into three groups : $v^{\|}, v^{\|, \pitchfork}$, and $v^{\pitchfork}$. The restrictions imposed on $f_{2}, g_{21}$, and $g_{22}$ after (4) imply that $\left(v^{\|}, v^{\|, \pitchfork}, v^{\pitchfork}\right)=(\star, \star, 0)$, where $\star$ represents arbitrary functions, renders $\Xi\left(S_{1} \cap U\right)$ locally invariant, i.e., $(\star, \star, 0)$ is a friend of $\Xi\left(S_{1} \cap U\right)$. Substituting $\xi=0$ and $v^{\pitchfork}=0$ in (3) the dynamics of (1) restricted to $\Xi\left(S_{1} \cap U\right)$ are

$$
\begin{aligned}
& \dot{\zeta}=f_{1}(\zeta, \mu, 0)+g_{11}(\zeta, \mu, 0) v^{\|}+g_{12}(\zeta, \mu, 0) v^{\|, \pitchfork} \\
& \dot{\mu}=A \mu+B v^{\|, \pitchfork} .
\end{aligned}
$$

The $\mu$-subsystem in (5) is linear and controllable and represents the dynamics of (1) restricted to $\Xi\left(S_{1} \cap U\right)$ and transversal to $\Xi\left(S_{2} \cap U\right)$. The control input $v^{\|, \pitchfork}$ can effectively be used to stabilize $S_{2} \cap U$ relative to $S_{1} \cap U$. Finally $\Xi\left(S_{2} \cap U\right)$ is controlled-invariant with friend $\left(v^{\|}, v^{\|, \pitchfork}, v^{\pitchfork}\right)=(\star, 0,0)$. The dynamics of (1) restricted to $\Xi\left(S_{2} \cap U\right)$ are

$$
\dot{\zeta}=f_{1}(\zeta, 0,0)+g_{11}(\zeta, 0,0) v^{\|} .
$$

Remark II.1. In (3) the $\mu$-subsystem is not feedback linearized. It only becomes linear when it evolves on $\Xi\left(S_{1} \cap U\right)$.

\section{PRELIMINARY RESUlTS}

In this section we investigate the problem of restricting the control-affine system (1) to nested sets $S_{1} \supset S_{2}$ satisfying Assumption 1. Due to space limitations the proofs of various results in this section are omitted. The interested reader can find these proofs in [8], [9].

Problem 2. Given the control system (1), nested sets $S_{1} \supset$ $S_{2}$ satisfying Assumption 1, and a point $\bar{x} \in S_{2}$, find, if possible, a diffeomorphism $\Xi: U \rightarrow \Xi(U) \subseteq \mathbb{R}^{s_{2}} \times \mathbb{R}^{s_{1}-s_{2}} \times$ $\mathbb{R}^{n-s_{1}}, x \mapsto(\zeta, \mu, \xi)$, and a regular feedback transformation $(\alpha, \beta), u \mapsto\left(v^{\|}, v^{\|, \pitchfork}, v^{\pitchfork}\right)$ valid in a neighbourhood $U \subseteq \mathbb{R}^{n}$ of $\bar{x}$, such that (1) is feedback equivalent to

$$
\begin{aligned}
& \dot{\zeta}=f_{1}+g_{11} v^{\|}+g_{12} v^{\|, \pitchfork}+g_{13} v^{\pitchfork} \\
& \dot{\mu}=f_{2}+g_{21} v^{\|}+g_{22} v^{\|, \pitchfork}+g_{23} v^{\pitchfork} \\
& \dot{\xi}=f_{3}+g_{31} v^{\|}+g_{32} v^{\|, \pitchfork}+g_{33} v^{\text {, }}
\end{aligned}
$$

where $f_{i}, g_{i j}$ for $i, j \in\{1,2,3\}$ are functions of $(\zeta, \mu, \xi)$,

$$
\begin{aligned}
& \Xi\left(S_{1} \cap U\right)=\{(\zeta, \mu, \xi) \in \Xi(U): \xi=0\}, \\
& \Xi\left(S_{2} \cap U\right)=\{(\zeta, \mu, \xi) \in \Xi(U): \xi=0, \mu=0\},
\end{aligned}
$$

$f_{3}(\zeta, \mu, 0)=0, g_{31}(\zeta, \mu, 0)=0, g_{32}(\zeta, \mu, 0)=0$, $f_{2}(\zeta, 0,0)=0$, and $g_{21}(\zeta, 0,0)=0$.

Like (3), the normal form (7) features two types of decomposition. However, unlike (3), we do not require the transversal dynamics to $S_{2}$, restricted to $S_{1}$ and transversal dynamics to $S_{1}$ be linear and controllable.

The normal form (7) is useful for understanding the interplay between the control vector fields $g_{1}, \cdots, g_{m}$ of (1) and the nested sets $S_{1} \supset S_{2}$. To understand this, first note that $g$ is partitioned into three sub-matrices corresponding to $v^{\|}, v^{\|, \pitchfork}$, and $v^{\pitchfork}$. The impositions on $g_{21}$ and $g_{31}$ mean that the columns of the matrix $\Xi_{\star}(g \beta)$ corresponding to 
$v^{\|}$are tangent to both $\Xi\left(S_{1} \cap U\right)$ and $\Xi\left(S_{2} \cap U\right)$. The requirement on $g_{32}$ implies that the columns corresponding to $v^{\|, \pitchfork}$ are tangent to $\Xi\left(S_{1} \cap U\right)$ but not $\Xi\left(S_{2} \cap U\right)$. Finally, the requirements on $f_{2}, f_{3}$, imply that the vector field $\Xi_{\star}(f+g \alpha)=\left(f_{1}, f_{2}, f_{3}\right)$ is tangent to both $\Xi\left(S_{1} \cap U\right)$ and $\Xi\left(S_{2} \cap U\right)$. Substituting $\xi=0$ and $v^{\pitchfork}=0$, the dynamics of (1) restricted to $\Xi\left(S_{1} \cap U\right)$ are

$$
\begin{aligned}
& \dot{\zeta}=f_{1}(\zeta, \mu, 0)+g_{11}(\zeta, \mu, 0) v^{\|}+g_{12}(\zeta, \mu, 0) v^{\|, \pitchfork} \\
& \dot{\mu}=f_{2}(\zeta, \mu, 0)+g_{21}(\zeta, \mu, 0) v^{\|}+g_{22}(\zeta, \mu, 0) v^{\|, \pitchfork} .
\end{aligned}
$$

The tangential dynamics on $S_{2}$ are the same as (6). The aforementioned partition of $g$ is closely related to the properties of the distributions

$$
\begin{aligned}
P & :=G_{0} \cap T S_{2} \\
Q & :=\left[G_{0} \cap T S_{2}\right]^{\perp} \cap\left[G_{0} \cap T S_{1}\right] \\
R & :=\left[G_{0} \cap T S_{1}\right]^{\perp} \cap G_{0}
\end{aligned}
$$

and the integer-valued functions $\nu, \rho: S_{2} \rightarrow \mathbb{Z}, \sigma: S_{1} \rightarrow \mathbb{Z}$

$$
\begin{aligned}
\nu(x) & :=\operatorname{dim}\left(T_{x} S_{2} \cap G_{0}(x)\right) \\
\rho(x) & :=\operatorname{dim}\left(T_{x} S_{1} \cap G_{0}(x)\right)-\nu(x) \\
\sigma(x) & :=\operatorname{dim}\left(T_{x} S_{1}+G_{0}(x)\right)-s_{1} .
\end{aligned}
$$

The values of (11) equal the dimensions of the distributions (10) and the sizes of the sub-matrices corresponding to $v^{\|}, v^{\|, \pitchfork}$, and $v^{\pitchfork}$ in (7).

Proposition III.1. For all $p \in S_{1}, q \in S_{2}, \operatorname{dim}(P(q))=$ $\nu(q), \operatorname{dim}(Q(q))=\rho(q)$ and $\operatorname{dim}(R(p))=\sigma(p)$.

Proof. The proof that $\operatorname{dim}(P(q))=\nu(q)$ is obvious from their definitions and is omitted. Next we have

$$
\begin{aligned}
& \operatorname{dim}(Q(q)) \\
& =n-\operatorname{dim}\left(G_{0}(q) \cap T_{q} S_{2}\right)+\operatorname{dim}\left(G_{0}(q) \cap T_{q} S_{1}\right) \\
& -\operatorname{dim}\left(\left[G_{0}(q) \cap T_{q} S_{2}\right]^{\perp}+\left[G_{0}(q) \cap T_{q} S_{1}\right]\right) \\
& =\operatorname{dim}\left(G_{0}(q) \cap T_{q} S_{1}\right)-\operatorname{dim}\left(G_{0}(q) \cap T_{q} S_{2}\right) \\
& =\rho(q) .
\end{aligned}
$$

Similar computations yield $\operatorname{dim}(R(p))=\sigma(p)$ on $S_{1}$.

Definition III.2. A point $\bar{x} \in S_{2}$ is a regular point of the distributions (10) if there exists an open set $V_{1} \subseteq S_{1}$ containing $\bar{x}$ such that for all $p \in V_{1}, q \in V_{1} \cap S_{2}$, the functions $\sigma(p), \nu(q), \rho(q)$ are constant.

The main result of this section can now be stated.

Theorem III.3. Problem 2 is solvable at $\bar{x} \in S_{2}$ if and only if $\bar{x}$ is a regular point of (10).

Before presenting the proof of Theorem III.3, we need some preliminary results whose proofs are omitted due to space limitations. The next proposition provides a computationally tractable way of checking the regularity of the distributions (10).

Proposition III.4. A point $\bar{x} \in S_{2}$ is a regular point of (10) if and only if $\operatorname{dim}\left(T_{x} S_{1} \cap G_{0}(x)\right)$ and $\operatorname{dim}\left(T_{x} S_{2} \cap G_{0}(x)\right)$ are constant in, respectively, open sets $V_{1} \subseteq S_{1}, V_{2} \subseteq S_{2}$ containing $\bar{x}$.

Remark III.5. It is easy to show that if any two of the functions in (11) are constant in an open subset of $S_{2}$, then the remaining function is also constant on this set. Furthermore, if $x$ is a regular point of (11) then $\nu(x)+\rho(x)+\sigma(x)=m$.

Proposition III.6. A point $\bar{x} \in S_{2}$ is a regular point of (10) if and only if there exists an open set $V_{1} \subseteq S_{1}$ containing $\bar{x}$ such that the distributions (10) are smooth and non-singular in $V_{1}$ and $V_{1} \cap S_{2}$.

Lemma III.7. The values of the functions (11) are invariant under coordinate and feedback transformation.

Lemma III.8. Let $S_{1} \supset S_{2}$ be two smooth embedded submanifolds of $\mathbb{R}^{n}$. For all $\bar{x} \in S_{2}$ there exists an open set $U \subseteq \mathbb{R}^{n}$ such that $S_{1}$ and $S_{2}$ are, respectively, $s_{1}$-slices and $s_{2}$-slices of $U$.

The tubular neighbourhood theorem [10, Theorem 10.19] states that every embedded submanifold $M$ of $\mathbb{R}^{n}$ has a tubular neighbourhood $\mathcal{N}(M)$. It follows [10, Proposition 10.20] that if $\mathcal{N}(M)$ is a tubular neighbourhood of an embedded submanifold $M \subset \mathbb{R}^{n}$, there exists a smooth retraction of $\mathcal{N}(M)$ onto $M$. In this paper we use a simpler, local version of these ideas.

Lemma III.9. Let $M \subset \mathbb{R}^{n}$ be an $m$-dimensional embedded submanifold of $\mathbb{R}^{n}$. Then, for every $x \in M$ there exist a neighbourhood $U$ of $x$ in $\mathbb{R}^{n}$ and a smooth retraction $r$ : $U \rightarrow M \cap U$.

Lemma III.10. Consider two sets $S_{1}$ and $S_{2}$ satisfying Assumption 1 and let $\bar{x} \in S_{2}$ be a regular point of (10). There exists an open set $U \subseteq \mathbb{R}^{n}$ containing $\bar{x}$ and a smooth feedback $\alpha: U \rightarrow \mathbb{R}^{m}$ such that $\left.(f+g \alpha)\right|_{S_{1} \cap U}$ is tangent to $S_{1} \cap U$ and $\left.(f+g \alpha)\right|_{S_{2} \cap U}$ is tangent to $S_{2} \cap U$.

Proof of Theorem III.3. Assume that Problem 2 is solvable at $\bar{x} \in S_{2}$. Then there exists a neighbourhood $U \subseteq \mathbb{R}^{n}$ containing $\bar{x}$, a feedback transformation $(\alpha, \beta)$ defined on $U$, and a diffeomorphism $\Xi \in \operatorname{Diff}(U)$ such that (1) is locally feedback equivalent to (7). Let $V_{2}:=S_{2} \cap U, V_{1}:=S_{1} \cap U$, denote by $(\zeta, \mu, 0)=\Xi(x)$ the image of a point $x \in V_{1}$ and by $(\zeta, 0,0)=\Xi(x)$ the image of a point $x \in V_{2}$ under the map $\Xi$, and let $\hat{g}:=\Xi_{\star}(g \beta), \hat{G}_{0}:=\operatorname{span}\left\{\hat{g}_{1}, \cdots, \hat{g}_{m}\right\}$. In $(\zeta, \mu, \xi)$-coordinates the value of $\sigma$ in (11b) at an arbitrary point $(\zeta, \mu, 0) \in \Xi\left(V_{1}\right)$ equals

$$
\begin{aligned}
\sigma(\zeta, \mu, 0) & =\operatorname{dim}\left(\operatorname{Im}\left[\begin{array}{cc}
I_{s_{1}} & \star \\
0 & g_{33}(\zeta, \mu, 0)
\end{array}\right]\right)-s_{1} \\
& =\operatorname{rank}\left(g_{33}(\zeta, \mu, 0)\right) .
\end{aligned}
$$

We now argue that $g_{33}$ has full column rank. The equality above implies that the number of columns in $g_{33}(\zeta, \mu, 0)$ is greater than or equal to $\sigma(\zeta, \mu, 0)$. Suppose, by way of contradiction, that $g_{33}$ has $\sigma(\zeta, \mu, 0)+1$ columns. Then, since 
there are $m$ inputs

$$
\operatorname{rank}\left[\begin{array}{cc}
g_{11}(\zeta, \mu, 0) & g_{12}(\zeta, \mu, 0) \\
g_{21}(\zeta, \mu, 0) & g_{22}(\zeta, \mu, 0)
\end{array}\right] \leq m-\sigma(\zeta, \mu, 0)-1 .
$$

But this means that $\operatorname{dim}\left(\hat{G}_{0}(\zeta, \mu, 0)\right) \leq m-1$ which is a contradiction since $(\alpha, \beta)$ is a regular feedback transformation and $\operatorname{dim}\left(G_{0}(x)\right)=m$. Thus $g_{33}(\zeta, \mu, 0)$ has full column rank. This shows that at an arbitrary point $(\zeta, \mu, 0)$, the integer function $\sigma$ is equal to the number of columns in $g_{33}$. Since $(\zeta, \mu, 0)$ is arbitrary, we conclude that $\sigma$ is constant on $\Xi\left(V_{1}\right)$.

Having shown that $g_{33}(\zeta, \mu, 0)$ has full column rank on $\Xi\left(V_{1}\right)$ it follows that $\nu(\zeta, 0,0)$ equals

$$
\begin{aligned}
& \operatorname{dim}\left(T_{(\zeta, 0,0)} \Xi\left(V_{2}\right) \cap \hat{G}_{0}(\zeta, 0,0)=\right. \\
& \operatorname{dim}\left(\operatorname{Im}\left[\begin{array}{c}
I_{s_{2}} \\
0
\end{array}\right] \cap \operatorname{Im}\left[\begin{array}{cc}
g_{11}(\zeta, 0,0) & g_{12}(\zeta, 0,0) \\
0 & g_{22}(\zeta, 0,0)
\end{array}\right]\right) .
\end{aligned}
$$

Additionally, since

$$
\begin{aligned}
& \operatorname{dim}\left(\left[\begin{array}{c}
I_{s_{2}} \\
0
\end{array}\right]+\operatorname{Im}\left[\begin{array}{cc}
g_{11}(\zeta, 0,0) & g_{12}(\zeta, 0,0) \\
0 & g_{22}(\zeta, 0,0)
\end{array}\right]\right)= \\
& \operatorname{rank}\left[\begin{array}{cc}
I_{s_{2}} & \star \\
0 & g_{22}(\zeta, 0,0)
\end{array}\right]
\end{aligned}
$$

we get that $\nu(\zeta, 0,0)=s_{2}+m-\sigma(\zeta, 0,0)-$ (rank $\left.g_{22}(\zeta, 0,0)+s_{2}\right)$. Using the above expression for $\nu$ and the identity, see Remark III.5, $\nu+\rho+\sigma=m$ at $(\zeta, 0,0)$ we obtain $\rho(\zeta, 0,0)=\operatorname{rank} g_{22}(\zeta, 0,0)$. Using the same reasoning as earlier, one can show $g_{22}(\zeta, 0,0)$ has full column rank. Thus, at $(\zeta, 0,0)$, the integer function $\rho$ is equal to the number of columns in $g_{22}$ which is constant at any point $(\zeta, 0,0) \in \Xi\left(V_{2}\right)$. Finally, if two of the functions in (11) are constant on $\Xi\left(V_{2}\right)$, then so is the third. By Lemma III.7 the values of (11) are invariant under feedback and coordinate transformation which shows that $\bar{x}$ is a regular point of (10).

Assume that $\bar{x} \in S_{2}$ is a regular point of (10). By Proposition III.6 the distribution $R$ in (10) is smooth and non-singular in a neighbourhood $V_{1} \subseteq S_{1}$ containing $\bar{x}$ and the distributions $P$ and $Q$ in (10) are smooth and nonsingular in $V_{2}=V_{1} \cap S_{2}$. As a result, there exist local generators $\hat{p}_{i}: V_{2} \rightarrow \mathbb{R}^{n}, i \in\{1, \cdots, \nu\}, \hat{q}_{i}: V_{2} \rightarrow \mathbb{R}^{n}$, $i \in\{1, \cdots, \rho\}$, and $\hat{r}_{i}: V_{1} \rightarrow \mathbb{R}^{n}, i \in\{1, \cdots, \sigma\}$ such that, for all $x \in V_{2} P(x)=\operatorname{span}\left\{\hat{p}_{1}, \cdots, \hat{p}_{\nu}\right\}(x)$ and $Q(x)=\operatorname{span}\left\{\hat{q}_{1}, \cdots, \hat{q}_{\rho}\right\}(x)$ and for all $x \in V_{1}$ $R(x)=\operatorname{span}\left\{\hat{r}_{1}, \cdots, \hat{r}_{\sigma}\right\}(x)$. Next, applying Lemma III.9 we introduce a retraction $r_{1}: U \rightarrow V_{1}$ of an open set $U \subseteq \mathbb{R}^{n}, \bar{x} \in U$, onto $V_{1}$ and a retraction $r_{2}: U \rightarrow V_{2}$ of an open set $U \subseteq \mathbb{R}^{n}, \bar{x} \in U$, onto $V_{2}$ and define

$$
\begin{aligned}
& p_{i}: U \rightarrow \mathbb{R}^{n} \\
& x \mapsto \hat{p}_{i} \circ r_{2}(x) \\
& i \in\{1, \cdots, \nu\} \\
& q_{i}: U \rightarrow \mathbb{R}^{n} \\
& x \mapsto \hat{q}_{i} \circ r_{2}(x) \\
& i \in\{1, \cdots, \rho\}
\end{aligned}
$$

$$
\begin{aligned}
& r_{i}: U \rightarrow \mathbb{R}^{n} \\
& x \mapsto \hat{r}_{i} \circ r_{1}(x)
\end{aligned} \quad i \in\{1, \cdots, \sigma\}
$$

so that the local generators of $P(x), Q(x)$, and $R(x)$ are now defined on $U$. We set up the following equations

$$
\begin{aligned}
& {\left[\begin{array}{lll}
p_{1} & \cdots & p_{\nu}
\end{array}\right]=\left[\begin{array}{lll}
g_{1} & \cdots & g_{m}
\end{array}\right] \beta_{1}} \\
& {\left[\begin{array}{lll}
q_{1} & \cdots & q_{\rho}
\end{array}\right]=\left[\begin{array}{lll}
g_{1} & \cdots & g_{m}
\end{array}\right] \beta_{2}} \\
& {\left[\begin{array}{llll}
r_{1} & \cdots & r_{\sigma}
\end{array}\right]=\left[\begin{array}{lll}
g_{1} & \cdots & g_{m}
\end{array}\right] \beta_{3}}
\end{aligned}
$$

where $\beta_{1}: U \rightarrow \mathbb{R}^{m \times \nu}, \beta_{2}: U \rightarrow \mathbb{R}^{m \times \rho}$, and $\beta_{3}: U \rightarrow$ $\mathbb{R}^{m \times \sigma}$ are unknown matrices. Since, $P \subseteq G_{0}$ and both are constant dimensional, by possibly shrinking $U$, there exists a unique smooth solution $\beta_{1}$ to (12a). Similarly, by shrinking $U$, we can find $\beta_{2}$ and $\beta_{3}$ in equations (12b) and (12c), respectively. Define $\left[\beta_{1} \beta_{2} \beta_{3}\right]=: \beta: U \rightarrow \mathrm{GL}(m, \mathbb{R})$.

Since $P(x), Q(x)$, and $R(x)$ span independent subspaces for each $x \in U$, the matrix $\beta$ is non-singular.

By Lemma III.10 there exists a feedback $\alpha: U \rightarrow$ $\mathbb{R}^{m}$ defined, without loss of generality, on $U$ such that $\left.(f+g \alpha)\right|_{S_{1} \cap U}$ is tangent to $V_{1}:=S_{1} \cap U$ and $\left.(f+g \alpha)\right|_{S_{2} \cap U}$ is tangent to $V_{2}:=S_{2} \cap U$. The pair $(\alpha, \beta)$ is the desired feedback transformation. Applying it to (1) yields

$\dot{x}=f(x)+g(x) \alpha(x)+g(x) \beta_{1}(x) v^{\|}+g(x) \beta_{2}(x) v^{\|, \pitchfork}+g(x) \beta_{3}(x) v^{\pitchfork}$

where $v^{\|} \in \mathbb{R}^{\nu}, v^{\|, \pitchfork} \in \mathbb{R}^{\rho}$, and $v^{\pitchfork} \in \mathbb{R}^{\sigma}$.

By Lemma III.10 the vector field $\left.(f(x)+g(x) \alpha(x))\right|_{V_{2}}$ is tangent to both $V_{1}$ and $V_{2}$. Columns of $\left.g \beta_{1}\right|_{V_{2}}$ are the local generators of $P$ thus are tangent to $V_{2}$. the columns of $\left.g \beta_{2}\right|_{V_{2}}$ are local generators of $Q$, so are tangent to $V_{1}$ and not $V_{2}$. Finally, columns of $\left.g \beta_{3}\right|_{V_{2}}$ are local generators of $R$, so are tangent to neither $V_{1}$ nor $V_{2}$. Select $\Xi$ to be the diffeomorphism from Lemma III.8. Applying the coordinate transformation $\Xi$ to (13) yields the desired normal form (7).

\section{SOlution to LOCAL NESTED TRANSVERSE FEEDBACK LINEARIZATION PROBLEM}

We are now ready to present the main result of this paper, necessary and sufficient conditions for Problem 1 to be solvable. It is evident that (3) is a refinement of (7) and thus the solvability of Problem 2 is a necessary condition for Problem 1 to be solvable.

Assumption 2. The point $\bar{x} \in S_{2}$ is a regular point of (10).

Assumption 2 implies, by Theorem III.3, that Problem 2 is solvable at $\bar{x}$. Therefore, there exists a regular feedback transformation $(\alpha, \beta)$ such that, control system (1) on a neighbourhood $U \subseteq \mathbb{R}^{n}$ of $\bar{x}$ writes as (13). Recall that $v^{\pitchfork}=0$ renders $S_{1} \cap U$ invariant; and the vector field $f+g \alpha$ and columns of $g \beta_{1}$ and columns of $g \beta_{2}$ are tangent to $S_{1} \cap U$. Thus, the restriction of (1) with $v^{\pitchfork}=0$ to $S_{1} \cap U$ is well-defined. We introduce the following shorthand notation

$f_{S_{1}}:=\left.(f+g \alpha)\right|_{S_{1} \cap U}, g_{S_{1}}:=\left.\left[g \beta_{1} g \beta_{2}\right]\right|_{S_{1} \cap U} v_{S_{1}}^{\|}:=\left(v^{\|}, v^{\|, \pitchfork}\right)$. 
Then, the dynamics restricted to $S_{1} \cap U$ are

$$
\dot{x}=f_{S_{1}}(x)+g_{S_{1}}(x) v_{S_{1}}^{\|} .
$$

Similar to (2), we associate to (14) a family of distribution $G_{i}^{\|}: S_{1} \cap U \rightarrow T\left(S_{1} \cap U\right) \subseteq T \mathbb{R}^{n}$

$G_{i}^{\|}(x):=\operatorname{span}\left\{a d_{f_{S_{1}}}^{j} g_{S_{1}, k}(x): 0 \leq j \leq i, 1 \leq k \leq \nu+\rho\right\}$

Theorem IV.1 (Main Result). Consider (1) and nested sets $S_{1} \supset S_{2}$ satisfying Assumption 1. Let $\bar{x} \in S_{2}$ and assume that the distributions $\operatorname{inv}\left(G_{i}^{\|}\right), \operatorname{inv}\left(G_{j}\right), i \in \mathbf{n}-\mathbf{s}_{\mathbf{1}}-\mathbf{1}$, $j \in \mathbf{s}_{\mathbf{1}}-\mathbf{s}_{\mathbf{2}}-\mathbf{1}$ are regular at $\bar{x} \in S_{2}$. Then, Problem 1 is solvable if and only if

(a) $\bar{x}$ is a regular point of (10)

(b) $\operatorname{dim}\left(T_{\bar{x}} S_{1}+G_{n-s_{1}-1}(\bar{x})\right)=n$

(c) There exist a neighbourhood $U$ of $\bar{x}$ in $\mathbb{R}^{n}$ such for all $i \in \mathbf{n}-\mathbf{s}_{\mathbf{1}}-\mathbf{1}$, for all $\left(x \in S_{1} \cap U\right)$,

$$
\operatorname{dim}\left(T_{x} S_{1}+G_{i}(x)\right)=\left(T_{x} S_{1}+\operatorname{inv}\left(G_{i}\right)(x)\right)=\text { const }
$$

(d) $\operatorname{dim}\left(T_{\bar{x}} S_{2}+G_{s_{1}-s_{2}-1}^{\|}(\bar{x})\right)=s_{1}$

(e) There exists an open set $U \subseteq \mathbb{R}^{n}$ containing $\bar{x}$ such that, for all $i \in \mathbf{s}_{\mathbf{1}}-\mathbf{s}_{\mathbf{2}}-\mathbf{1}$, for all $x \in S_{2} \cap U$

$$
\operatorname{dim}\left(T_{x} S_{2}+G_{i}^{\|}(x)\right)=\left(T_{x} S_{2}+\operatorname{inv}\left(G_{i}^{\|}(x)\right)\right)=\text { const }
$$

Proof. Suppose that Problem 1 is solvable at $\bar{x} \in S_{2}$. Then Problem 2 is solvable since the normal form (3) is a refinement of the normal form (7). Thus, $\bar{x}$ is a regular point of (10) and condition (a) holds. As a result, the assumption requiring $\operatorname{inv}\left(G_{i}^{\|}\right), i \in \mathbf{s}_{\mathbf{1}}-\mathbf{s}_{\mathbf{2}}-\mathbf{1}$ be regular at $\bar{x}$ is a valid assumption. Moreover, since Problem 1 is solvable at $\bar{x} \in S_{2}$ there exists a neighbourhood $U \subseteq \mathbb{R}^{n}$ of $\bar{x}$, a coordinate transformation $\Xi \in \operatorname{Diff}(U)$, and a feedback transformation $(\alpha, \beta)$ such that (1) is feedback equivalent to (3) in $U$. Define $V_{1}:=\Xi\left(S_{1} \cap U\right)$ and $V_{2}:=\Xi\left(S_{2} \cap U\right)$. The system dynamics restricted to $V_{1}$ are given in (5), and any point in $V_{1}$ and $V_{2}$ is represented by $(\zeta, \mu)$ and $(\zeta, 0)$, respectively. In transformed coordinates we have

$$
\begin{aligned}
& \left(\forall(\zeta, 0) \in V_{2}\right),\left(\forall i \in \mathbf{s}_{\mathbf{1}}-\mathbf{s}_{\mathbf{2}}\right), T_{(\zeta, 0)} V_{2}+G_{i}^{\|}(\zeta, 0)= \\
& \operatorname{Im}\left(\left[\begin{array}{ccccc}
I_{s_{2}} & \star & \star & \cdots & \star \\
0_{r-s_{2} \times s_{2}} & B & A B & \cdots & A^{i} B
\end{array}\right]\right)
\end{aligned}
$$

which shows that the dimension of $T_{(\zeta, 0)} V_{2}+G_{i}^{\|}(\zeta, 0)$ is $s_{2}+\operatorname{rank}\left(\left[\begin{array}{ccc}B & \cdots & A^{i} B\end{array}\right]\right)$. Since the pair $(A, B)$ is controllable, $\operatorname{rank}\left(\left[\begin{array}{ccc}B & \cdots & A^{s_{1}-s_{2}-1} B\end{array}\right]\right)=s_{1}-s_{2}$; thus $\operatorname{dim}\left(T_{(\zeta, 0)} V_{2}+G_{s_{1}-s_{2}-1}^{\|}(\zeta, 0)\right)=s_{1}$. Since condition $(d)$ is invariant under coordinate and feedback transformations it follows that condition $(d)$ holds in original coordinates as well.

In $V_{1}$, consider the collection of constant distributions $\Delta_{i}^{\|}, i \in \mathbf{s}_{\mathbf{1}}-\mathbf{s}_{\mathbf{2}}$ given by

$$
\Delta_{i}^{\|}:=\operatorname{Im}\left(I_{s_{2}} \oplus\left[\begin{array}{lll}
B & \cdots & A^{i} B
\end{array}\right]\right) .
$$

At each $(\zeta, 0) \in V_{2}, \Delta_{i}^{\|}(\zeta, 0)=T_{(\zeta, 0)} V_{2}+G_{i}^{\|}(\zeta, 0)$; thus, $G_{i}^{\|}(\zeta, 0) \subseteq \Delta_{i}^{\|}$. Furthermore, since each $\Delta_{i}^{\|}$is (trivially) involutive, it follows that $\operatorname{inv}\left(G_{i}^{\|}(\zeta, 0)\right) \subseteq \Delta_{i}^{\|}$. This shows that for all $i \in \mathbf{s}_{\mathbf{1}}-\mathbf{s}_{\mathbf{2}}$

$$
T V_{2}+\operatorname{inv}\left(G_{i}^{\|}(\zeta, 0)\right) \subseteq \Delta_{i}^{\|}(\zeta, 0)=T V_{2}+G_{i}^{\|}(\zeta, 0) .
$$

On the other hand $T V_{2}+G_{i}^{\|}(\zeta, 0) \subseteq T V_{2}+\operatorname{inv}\left(G_{i}^{\|}(\zeta, 0)\right)$ always holds which shows that $\Delta_{i}^{\|}(\zeta, 0)=T V_{2}+G_{i}^{\|}(\zeta, 0)=$ $T V_{2}+\operatorname{inv}\left(G_{i}^{\|}(\zeta, 0)\right)$. Condition $(e)$ is invariant under coordinate and feedback transformations; thus it holds in original coordinates. The proof of the necessity of conditions (b) and (c) is easily checked in transformed coordinates using analogous arguments.

Conversely, By [7, Theorem 3.2], since conditions (b) and (c) hold, there exist a neighbourhood $U$ of the point $\bar{x}$, a diffeomorphism $\Xi_{1}: U \rightarrow \Xi_{1}(U) \subset \mathbb{R}^{s_{1}} \times \mathbb{R}^{n-s_{1}}$ and a regular feedback transformation $\left(\alpha_{1}, \beta_{1}\right)$ such that system (1), on $U$, is feedback equivalent to

$$
\begin{aligned}
& \dot{\eta}=f_{0}(\eta, \xi)+g^{\|}(\eta, \xi) v_{1}+g^{\pitchfork}(\eta, \xi) v_{2} \\
& \dot{\xi}=E \xi+F v_{2}
\end{aligned}
$$

where the pair $(E, F)$ is controllable and $\Xi_{1}\left(S_{1} \cap U\right)=$ $\left\{(\eta, \xi) \in \Xi_{1}(U): \xi=0\right\}$. Since condition (a) hold Problem 2 is solvable. Thus, $v_{1} \in \mathbb{R}^{\nu+\rho}, v_{2} \in \mathbb{R}^{\sigma}$.

Let $\pi_{1}: \mathbb{R}^{n} \rightarrow \mathbb{R}^{s_{1}}$ be the projection to the first $s_{1}$ factors. Let $\bar{V}_{1}:=\pi_{1} \circ \Xi_{1}\left(S_{1} \cap U\right)$ and $\psi=\left.\pi_{1} \circ \Xi_{1}\right|_{S_{1} \cap U}: S_{1} \cap$ $U \rightarrow \bar{V}_{1}$. By [10, Theorem 8.2] $S_{1}$ is a smooth manifold of dimension $s_{1}$ and $\left(S_{1} \cap U, \psi\right)$ is a coordinate chart. Define $\bar{V}_{2}:=\pi_{1} \circ \Xi_{1}\left(S_{2} \cap U\right)$. Since $\bar{V}_{2}$ is diffeomorphic to $S_{2} \cap U$ it is an embedded submanifold of $\bar{V}_{1}$. In this coordinate chart the restricted dynamics in (14) writes as

$$
\dot{\eta}=f_{0}(\eta, 0)+g^{\|}(\eta, 0) v_{1} .
$$

By conditions (a) Problem 2 is solvable. Therefore, the assumption that $\operatorname{inv}\left(G_{i}^{\|}\right), i \in \boldsymbol{n}-\boldsymbol{s}_{\mathbf{1}}-\mathbf{1}$ are regular at $\bar{x}$ is well-posed. Since conditions (d) and (e) hold all the assumptions and conditions of [7, Theorem 3.2] for (17) with respect to $\bar{V}_{2}$ at $\bar{\eta}:=\pi_{1} \circ \Xi_{1}(\bar{x})$ hold. Therefore, by possibly shrinking $\bar{V}_{1}$ (and hence $U$ ), there exists a coordinate transformation $\Xi_{2}: \bar{V}_{1} \rightarrow \Xi_{2}\left(\bar{V}_{1}\right) \subseteq \mathbb{R}^{s_{2}} \times \mathbb{R}^{s_{1}-s_{2}}$, and a regular feedback transformation $\left(\bar{\alpha}_{2}, \bar{\beta}_{2}\right)$, with $\bar{\alpha}_{2}: \bar{V}_{1} \rightarrow \mathbb{R}^{\nu+\rho}$ and $\bar{\beta}_{2}: \bar{V}_{1} \rightarrow \mathrm{GL}(\nu+\rho, \mathbb{R})$, such that (17) is feedback equivalent to

$$
\begin{aligned}
& \dot{\zeta}=f_{1}(\zeta, \mu)+g_{11}(\zeta, \mu) v^{\|}+g_{12}(\zeta, \mu) v^{\|, \pitchfork} \\
& \dot{\mu}=A \mu+B v^{\|, \pitchfork} .
\end{aligned}
$$

Let $\pi_{2}: \mathbb{R}^{n} \rightarrow \mathbb{R}^{n-s_{1}}$ be the projection to the last $n-s_{1}$ factors. We construct a function $\Xi: U \subseteq \mathbb{R}^{n} \rightarrow \Xi(U) \subseteq$ $\mathbb{R}^{s_{2}} \times \mathbb{R}^{s_{1}-s_{2}} \times \mathbb{R}^{n-s_{1}}$ as follows

$$
\Xi:=\left(\Xi_{2} \circ \pi_{1} \circ \Xi_{1}\right) \times\left(\pi_{2} \circ \Xi_{1}\right)=\left[\begin{array}{c}
\Xi_{2} \circ \pi_{1} \\
\pi_{2}
\end{array}\right] \circ \Xi_{1} .
$$

The function $\Xi$ is a well-defined diffeomorphism since at $\bar{x}$ $\operatorname{det}(\mathrm{d} \Xi)=\operatorname{det}\left(\left[\begin{array}{cc}\mathrm{d} \Xi_{2} & 0_{s_{1} \times n-s_{1}} \\ 0_{n-s_{1} \times s_{1}} & I_{n-s_{1}}\end{array}\right]\right) \operatorname{det}\left(\mathrm{d} \Xi_{1}\right) \neq 0$. 
Therefore, by the inverse function theorem [10, Theorem 7.6], it is a valid coordinate transformation in a neighbourhood of $\bar{x}$, without loss of generality $U$. In order to construct the feedback transformation we define

$\alpha_{2}:=\left[\begin{array}{c}\bar{\alpha}_{2} \circ \pi_{1} \circ \Xi_{1} \\ 0_{\sigma}\end{array}\right], \quad \beta_{2}:=\left[\begin{array}{cc}\bar{\beta}_{2} \circ \pi_{1} \circ \Xi_{1} & 0_{(\nu+\rho) \times \sigma} \\ 0_{\sigma \times(\nu+\rho)} & I_{\sigma}\end{array}\right]$,

where $\alpha_{2}: U \subseteq \mathbb{R}^{n} \rightarrow \mathbb{R}^{m}$ and $\beta: U \subseteq \rightarrow \mathrm{GL}(m, \mathbb{R})$. The feedback transformation $(\alpha, \beta):=\left(\alpha_{1}+\beta_{1} \alpha_{2}, \beta_{1} \beta_{2}\right)$ and $\Xi \in \operatorname{Diff}(U)$ solve Problem 1 .

Example 1. Consider a system (1) with $m=2$,

$$
f(x)=\left[\begin{array}{c}
-x_{1} \\
-x_{2}-x_{5}-x_{5}\left(-x_{1}{ }^{2}+x_{2}+x_{3}\right) \\
x_{2}+x_{5}+x_{5}\left(-x_{1}{ }^{2}+x_{2}+x_{3}\right)-2 x_{1}{ }^{2} \\
x_{2}+x_{3}-x_{4}+x_{5}+x_{5}\left(-x_{1}{ }^{2}+x_{2}+x_{3}\right)-2 x_{1}{ }^{2} \\
x_{4}-x_{3}
\end{array}\right]
$$

$g_{1}(x)=\left(0,0,-1,-1, x_{2}\right), g_{2}(x)=(0,1,-1,-2,0)$, sets $S_{1}=\left\{x \in \mathbb{R}^{5}: x_{1}^{2}-x_{2}-x_{3}=0\right\}$ and $S_{2}=$ $\left\{x \in S_{1}: x_{2}=x_{3}-x_{4}=x_{5}=0\right\}$, and a point $\bar{x}=$ $(1,0,1,1,1)$. The objective is to solve Problem 1 at $\bar{x}$. Thus, we check the conditions of Theorem IV.1. It is easy to show that

$$
T_{x} S_{1}=\operatorname{Im}\left[\begin{array}{cccc}
1 & 0 & 0 & 0 \\
2 x_{1} & 1 & 0 & 0 \\
0 & -1 & 0 & 0 \\
0 & 0 & 1 & 0 \\
0 & 0 & 0 & 1
\end{array}\right], \quad T_{x} S_{2}=\operatorname{Im}\left[\begin{array}{c}
1 \\
0 \\
2 x_{1} \\
2 x_{1} \\
0
\end{array}\right]
$$

We compute for all $x \in \mathbb{R}^{n} \nu(x)=0$ and $\rho(x)=1$. Since the constancy of any two functions in (11) implies the constancy of the third, $\bar{x}$ is a regular point of (10) and condition (a) of Theorem IV.1 hold.

Condition (b) of Theorem IV.1 holds since $\operatorname{dim}\left(T_{\bar{x}} S_{1}+\right.$ $\left.G_{0}(\bar{x})\right)=5$. Condition (c) of Theorem IV.1 holds since $\operatorname{dim}\left(T_{x} S_{1}+G_{0}(x)\right)=\operatorname{dim}\left(T_{x} S_{1}+\operatorname{inv}\left(G_{0}(x)\right)\right)=5$ for all $x \in \mathbb{R}^{5}$.

In order to check conditions (d) and (e) of Theorem IV.1 we need to find (14). Following the proof of Theorem III.3 we find the restricted dynamics as

$$
\dot{x}=\left[\begin{array}{c}
-x_{1} \\
-x_{2}-x_{5} \\
x_{2}+x_{5}-2 x_{1}{ }^{2} \\
x_{2}+x_{3}-x_{4}+x_{5}-2 x_{1}{ }^{2} \\
x_{4}-x_{3}
\end{array}\right]+\left[\begin{array}{c}
0 \\
1 \\
-1 \\
-2 \\
0
\end{array}\right] v^{\|, \pitchfork} .
$$

Condition (d) of Theorem IV.1 is satisfied since, at the point $\bar{x}, \operatorname{dim}\left(T_{\bar{x}} S_{2}+G_{2}^{\|}(\bar{x})\right)=4$. Moreover, since $G_{0}^{\|}$ contains a single vector it is involutive and condition (e) of Theorem IV.1 hold. Thus Problem 1 is solvable. Following the proof of Theorem IV.1 we find the following feedback transformation and coordinate transformation

$$
\begin{gathered}
(\alpha, \beta)=\left(\left[\begin{array}{l}
0 \\
0
\end{array}\right],\left[\begin{array}{ll}
0 & 1 \\
1 & 0
\end{array}\right]\right), \\
\Xi=\left(x_{1}, x_{2}, x_{5}, x_{3}-x_{4}, x_{1}^{2}-x_{2}-x_{3}\right) .
\end{gathered}
$$

The given control system is feedback equivalent on $\mathbb{R}^{5}$ to

$$
\begin{aligned}
& \dot{\zeta}=-\zeta \\
& \dot{\mu}_{1}=-\mu_{1}-\mu_{2}+\mu_{2} \xi+v^{\|, \pitchfork} \\
& \dot{\mu}_{2}=-\mu_{3}+\mu_{1} v^{\pitchfork} \\
& \dot{\mu}_{3}=-\mu_{3}+v^{\|, \pitchfork} \\
& \dot{\xi}=v^{\pitchfork} .
\end{aligned}
$$

One can verify the second condition of [7, Theorem 3.2] does not hold for $S_{2}$ since for all $x \in \mathbb{R}^{n} \operatorname{dim}\left(T_{x} S_{2}+G_{0}\right) \neq$ $\operatorname{dim}\left(T_{x} S_{2}+\operatorname{inv}\left(G_{0}\right)\right)$. This implies that one cannot make the $\mu$-subsystem linear and decoupled. Thus the class of systems which is feedback equivalent to (3) is strictly larger than the class of systems for which the dynamics transversal to both $S_{1}$ and $S_{2}$ can be transversally feedback linearized.

\section{Conclusions}

We have determined necessary and sufficient conditions under which a nonlinear control-affine system, with two nested, controlled invariant, embedded submanifolds in its state space, is locally feedback equivalent to a system whose dynamics transversal to the larger submanifold, and dynamics restricted to the larger submanifold and transversal to the nested submanifold are linear and controllable. Future research entails posing global versions of Problems 1 and 2 that would be valid in a tubular neighbourhood of the larger set.

\section{REFERENCES}

[1] Mohamed I El-Hawwary and Manfredi Maggiore. Reduction theorems for stability of closed sets with application to backstepping control design. Automatica, 49(1):214-222, 2013.

[2] A. Doosthoseini and C. Nielsen. Coordinated path following for unicycles: A nested invariant sets approach. Automatica, 60:17-29, October 2015.

[3] P Seibert and JS Florio. On the reduction to a subspace of stability properties of systems in metric spaces. Annali di Matematica pura ed applicata, 169(1):291-320, 1995.

[4] A. J. Krener. On the equivalence of control systems and the linearization of nonlinear systems. SIAM Journal on Control and Optimization, 11(4):670-676, 1973.

[5] R. W. Brockett. Feedback invariants for nonlinear systems. In Proceedings of the IFAC World Congress, pages 1115-1120, Helsinki, 1978.

[6] A. Banaszuk and J. Hauser. Feedback linearization of transverse dynamics for periodic orbits. Systems \& Control Letters, 26:95-105, 1995.

[7] C. Nielsen and M. Maggiore. On local transverse feedback linearization. SIAM Journal on Control and Optimization, 47(5):2227-2250, 2008.

[8] A. Doosthoseini and C. Nielsen. Local nested transverse feedback linearization. Mathematics of Control, Signals, and Systems, 2015. Accepted.

[9] A. Doosthoseini. Coordinated path following: A nested invariant sets approach. $\mathrm{PhD}$ thesis, University of Waterloo, 2015. hdl. handle. net $/ 10012 / 9365$.

[10] J. M. Lee. Introduction to smooth manifolds, volume 218 of graduate texts in mathematics. Springer-Verlag, New York, 2003. 